\title{
Pseudoexfoliation and Opacification of Intraocular Lenses
}

\author{
Maria Milia $^{a}$ Aris Konstantopoulos $^{\mathrm{b}, \mathrm{c}}$ Panagiotis Stavrakas $^{\mathrm{d}}$ \\ Abbas Toufeeq ${ }^{\mathrm{e}}$ \\ ${ }^{a}$ Western Eye Hospital, London, ${ }^{b}$ Eye Unit, Stoke Mandeville Hospital, Aylesbury, \\ and 'Southampton Eye Unit, Southampton General Hospital, Southampton, UK; \\ ${ }^{\mathrm{d}}$ Attikon University Hospital, Athens, Greece; ${ }^{\mathrm{e}}$ Eye Department, Wycombe Hospital, \\ High Wycombe, UK
}

\section{Key Words}

Pseudoexfoliation · Opacification · Intraocular lens · Spoke-like pattern

\begin{abstract}
Purpose: To report two cases of spoke-like intraocular lens (IOL) opacification that resembles pseudoexfoliation of the crystalline lens.

Methods: Case series presentation.

Results: Patient 1 developed a circle of spoke-like opacification on the anterior IOL surface 7 years after phacoemulsification. Patient 2 had paracentral anterior surface IOL opacification 18 months after cataract extraction and clinical pseudoexfoliation in the fellow eye.

Conclusion: Spoke-like anterior IOL opacification should raise the suspicion of pseudoexfoliation syndrome.
\end{abstract}

\section{Introduction}

Intraocular lens (IOL) opacification is a rare but well-recognised complication following cataract surgery. Various types have been described and these are primarily caused by surface and intralenticular deposition of material [1,2]. A rarer spoke-like opacification pattern on the anterior IOL surface has been described by Bahadur and Masket [3] as 'pseudo-pseudoexfoliation'. Here, we describe two cases with a spoke-like opacification pattern, one with clinical pseudoexfoliation, the other with early exfoliation material on the IOL. 


\section{Case Reports}

Case 1

A 70-year-old female patient presented to our hospital 3 years following an uneventful left phacoemulsification. She was asymptomatic, with a Snellen best-corrected visual acuity (BCVA) of 6/9. Slit-lamp examination showed a thin semitransparent membrane on the anterior surface of the IOL. Intraocular pressure (IOP) was normal. She had had in-the-bag implantation of a plate-haptic hydrophilic IOL. Defocused application of YAG laser to the surface membrane and posterior capsulotomy were performed. Four years later, the patient remained asymptomatic, but slit-lamp examination showed that a circular spoke-like opacification, extending radially from the pupillary zone to the margins of the capsulorhexis (fig. 1), had developed on the surface of the IOL.

Case 2

A 76-year-old woman underwent uneventful right phacoemulsification with in-the-bag implantation of a plate-haptic hydrophilic lens, resulting in a BCVA of 6/9. The patient was reviewed 18 months later for cataract in the left eye, and ocular examination showed the presence of pseudoexfoliation in this eye. The IOP was normal in both eyes. It was noted that the IOL in the right eye showed paracentral surface opacification with a spoke-like pattern similar to Case 1 (fig. 2). The patient's IOP was monitored and she underwent YAG laser posterior capsulotomy in the right eye 8 months later. She is currently asymptomatic with a BCVA of $6 / 9$.

\section{Results}

We present two cases of spoke-like IOL opacification that resembles pseudoexfoliation of the crystalline lens. One patient had clinical pseudoexfoliation in the fellow eye, and the other had early exfoliation material on the affected IOL.

\section{Discussion}

Pseudoexfoliation involves the production of exfoliation fibres by pre-equatorial lens epithelial, non-pigmented ciliary epithelial and corneal endothelial cells, which are distributed by the aqueous humour to the central anterior lens capsule, zonules, anterior hyaloid surface and artificial lenses [4]. It is often forgotten that a precursor material is initially deposited on the lens surface, producing a homogeneous groundglass or matte appearance [5]. The early membrane that was present on the IOL surface in Case 1 may represent early exfoliation material. The classical appearance is then created by a thickening of this material and rubbing of the iris during pupillary movement on the surface of the lens.

Central IOL opacification may be related to local supersaturation with octacalcium phosphate, a process facilitated by surface hydroxyl groups of the polyacrylic materials [6]. The patterns of deposition in our two cases suggest that exfoliation material may deposit on the anterior IOL surface by a process similar to that in phakic pseudoexfoliation. Both patients had acrylic one-piece plate-haptic IOLs, with no posterior angulation of the optic. The lack of posterior angulation may facilitate the rubbing of the iris against the material deposited on the IOL surface, resulting in the spoke-like pattern. Interestingly, the case by Bahadur and Masket [3] also involved the use of a plate-haptic Collamer IOL. In general, hydrophilic acrylic IOLs have proven to have high uveal biocompatibility, inducing less inflammatory response than hydrophobic and silicone lenses [6]. The anterior surface of the artificial IOL is less 
curved and more posterior than that of the crystalline lens. As a result, the point of contact between the iris and the IOL is less peripheral than is the case with the crystalline lens. This may produce a spoke-like pattern that extends more centrally than on the surface of the crystalline lens.

\section{Conclusion}

In conclusion, the presence of a spoke-like pattern on the anterior IOL surface, especially on IOLs of plate-haptic design, should raise the suspicion of pseudoexfoliation. The presence of glaucoma in the case by Bahadur and Masket [3] highlights this issue. It may be advisable that such patients have regular IOP monitoring.

\section{Disclosure Statement}

The authors have no proprietary interest.

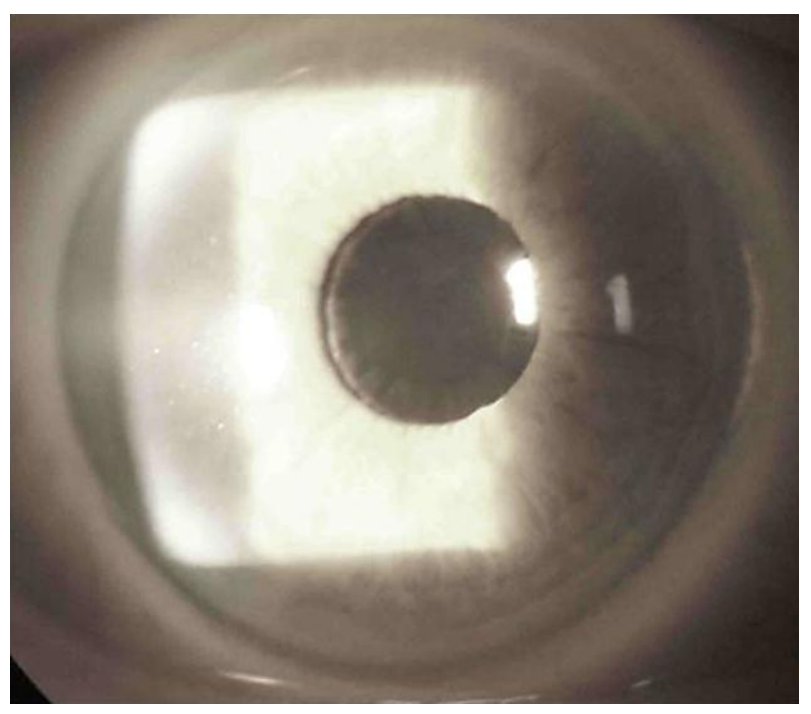

Fig. 1. Image demonstrating the spoke-like pattern on the anterior IOL surface. 


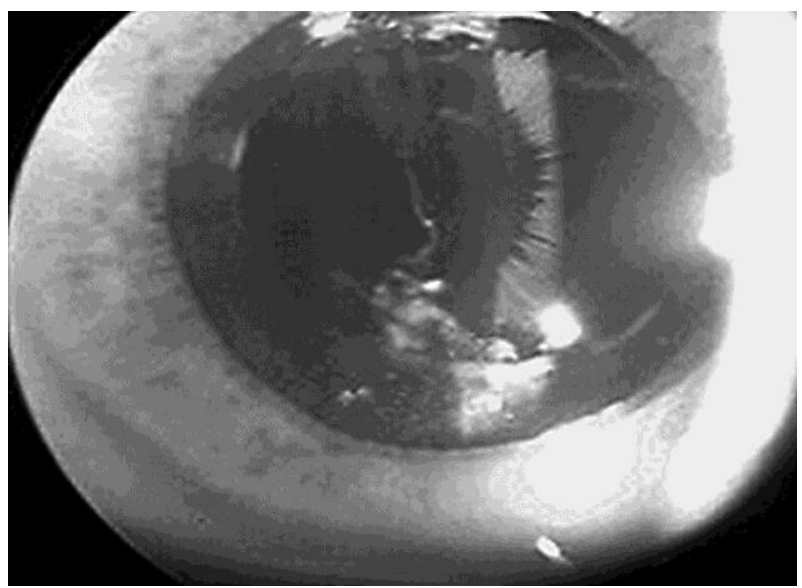

Fig. 2. Paracentral IOL surface opacification with a spoke-like pattern.

\section{References}

$\checkmark 1$ Neuhann IM, Werner L, Izak AM, Pandey SK, Kleinmann G, Mamalis N, Neuhann TF, Apple DJ: Late postoperative opacification of a hydrophilic acrylic (hydrogel) intraocular lens: a clinicopathological analysis of 106 explants. Ophthalmology 2004;111:2094-2101.

-2 Gartaganis SP, Kanellopoulou DG, Mela EK, Panteli VS, Koutsoukos PG: Opacification of hydrophilic acrylic intraocular lens attributable to calcification: investigation on mechanism. Am J Ophthalmol 2008;146:395-403.

-3 Bahadur GG, Masket S: Pseudophakia with pseudo-pseudoexfoliation. J Cataract Refract Surg 2007;33:1827-1828.

4 Ritch R, Schlötzer-Schrehardt U: Exfoliation syndrome. Surv Ophthalmol 2001;45:265-315.

-5 Tetsumoto K, Schlötzer-Schrehardt U, Küchle M, Dörfler S, Naumann GO: Precapsular layer of the anterior lens capsule in early pseudoexfoliation syndrome. Graefes Arch Clin Exp Ophthalmol 1992;230:252-257.

-6 Richter-Mueksch S, Kahraman G, Amon M, Schild-Burggasser G, Schauersberger J, Abela-Formanek C: Uveal and capsular biocompatibility after implantation of sharp-edged hydrophilic acrylic, hydrophobic acrylic, and silicone intraocular lenses in eyes with pseudoexfoliation syndrome. J Cataract Refract Surg 2007;33:1414-1418. 